\title{
Stadt, Ungleichheit und Diversität ${ }^{1}$
}

Mit dem Darmstädter Ansatz von der Eigenlogik der Städte wird nicht weniger beansprucht als eine Neugründung der Stadtsoziologie: eine "Grundlegung der Soziologie der Städte, welche um die Begriffe Eigenlogik, Doxa und Habitus gewebt ist «.$^{2}$ Mit der zentralen Kategorie der Eigenlogik »sind die verborgenen Strukturen der Städte als vor Ort eingespielte, zumeist stillschweigend wirksame Prozesse der Sinnkonstitution gemeint $«{ }^{3}$ Diese verborgenen Strukturen seien allumfassend, sie "webten " sich » in die für die Lebenspraxis konstitutiven Gegenstände hinein, in den menschlichen Körper (Habitus), in die Materialität der Wohnungen, Straßen, Zentrumsbildung, in die kulturelle Praxis, in die Redeweisen, in die emotionale Besetzung einer Stadt, in die politische Praxis, die wirtschaftliche Potenz, in die Marketingstrategien und so weiter «. ${ }^{4}$ Sie durchziehen »die Stadt wie ein Rückgrat [...] in allen sozialen Gruppen [...] und in der Organisation des öffentlichen Lebens «. ${ }^{5}$

Der Gegenstand einer wissenschaftlichen Disziplin ist immer doppelt konstituiert. Einmal durch die Disziplin, zum anderen durch die Wirklichkeit: Was in der wissenschaftlichen Community als die besonderen Fragestellungen, Erkenntnisinteressen, Theorien und Methoden etabliert ist, das definiert jenen Teil der Realität, der zum Gegenstand einer Disziplin werden kann. Wissenschaftliche Disziplinen sind wie Scheinwerfer, in deren Lichtkreis ein Ausschnitt der Wirklichkeit sichtbar wird. Aber die Wirklichkeit muss dieser Definition auch entgegenkommen, das heißt sie muss in diesem Lichtkreis auch in einer Weise sichtbar werden, wie es die theoretischen und methodischen Instrumente der jeweiligen Disziplin unterstellen. Mobilitätsforscher beispielsweise hätten wenig Freude am Studium der Bäume, es sei denn, diese könnten laufen wie zu jener Zeit, von der Tolkien im Herrn der Ringe erzählt. Und darin ist eine dritte Schwierigkeit bei der Definition eines wissenschaftlichen Gegenstands angedeutet, die vor allem für die Soziologie bedeutsam ist: Ihr Gegenstand ist zeitlichem Wandel unterworfen. Das aber bedeutet, dass sich das Gültigkeitsproblem, die

1 Der Beitrag ist die stark erweiterte und überarbeitete Fassung meines Vortrags in der Adhoc-Gruppe »Eigenlogik der Städte kontrovers: Zum aktuellen Perspektivenstreit in der Stadtsoziologie« auf dem Soziologentag 2012. Ich danke Thomas Krämer-Badoni, Michael Schumann und Uwe-Jens Walther für ihre hilfreiche Kritik einer ersten Version dieses Textes. Für eine ausführliche kritische Auseinandersetzung mit dem EigenlogikAnsatz vgl. auch Kemper, Vogelpohl 2011.

2 Löw 2010, S. 19.

3 Ebd.

4 Ebd., S. 77 f.

5 Löw 2008, S. 37. 
Frage, ob die Disziplin auch wirklich das erfassen kann, was sie am Gegenstand erfassen soll, immer von Neuem stellt, da im Zuge des sozialen Wandels die soziale Wirklichkeit aus den Kategorien, mit denen die Soziologie sie zu erfassen versucht, auswandert. Max Webers Definition von Stadt als einer revolutionären gesellschaftlichen Formation war gültig für die europäische Stadt des Mittelalters, aber sie ist es nicht mehr, weshalb es wenig Ertrag verspricht, mit Webers Definition heutige Städte zu erforschen. Deshalb ist der Versuch einer Neugründung der Stadtsoziologie doppelt zu befragen: zum einen, inwieweit er anschlussfähig ist an die zentralen Fragestellungen und Erkenntnisinteressen, die die Soziologie von anderen wissenschaftlichen Disziplinen unterscheidet, zum anderen, inwieweit damit Annahmen über die Wirklichkeit verbunden sind, die im Lichte bisherigen Wissens zumindest Plausibilität beanspruchen können.

\section{Was soll mit dem Darmstädter Ansatz anders werden?}

Die erste Kritik, mit der der Ansatz der Eigenlogik begründet wird, hält der bisherigen Stadtforschung vor, sie habe keinen Begriff von Stadt. Damit wird eine alte Frage wieder aufgenommen: die nach der Stadt als eigenständigem Gegenstand der Soziologie. Das ist in der Tat eine nicht befriedigend gelöste Frage, soweit darunter ein über die Grenzen der Epochen und der Kulturen hinweg gültiger Begriff von Stadt verstanden werden soll, wie ihn Louis Wirth und die Chicagoer Schule formuliert haben. Es ist aber eine Grundannahme der Stadtforschung, dass ein solcher allgemeiner Begriff von Stadt soziologisch leer bleiben muss. Friedrich Engels hatte in seiner Schrift » Die Lage der arbeitenden Klasse in England « ${ }^{6}$ eine ganz bestimmte Stadt vor Augen, nämlich das Manchester der ersten Hälfte des 19. Jahrhunderts, und er hat diese Stadt als eine ganz bestimmte Form von Stadt beschrieben, nämlich als die Stadt einer Klassengesellschaft. Hans Paul Bahrdt ${ }^{7}$ hat die Polarität von Öffentlichkeit und Privatheit zum Kern seiner Definition von Stadt gemacht. Auch er hat damit keine allgemeingültige Definition von Stadt vorgelegt, sondern die einer sehr spezifischen historischen Formation: der Stadt der bürgerlichen Gesellschaft. Stadt ist eine jeweils historisch besondere soziale Tatsache, die eine jeweils besondere räumliche Gestalt angenommen hat. Wenn daher von Stadt die Rede ist, dann kann das stets nur in Bezug auf eine bestimmte gesellschaftliche Formation sein. »Eine Stadt ist, was eine historische [...] Gesellschaft sie zu sein bestimmt. « ${ }^{8}$ Gegenstand der Stadtforschung im deutschen Sprachraum ist weit überwiegend die europäische Stadt des 19. und 20. Jahrhunderts, und das heißt die Stadt der bürgerlichen Gesellschaft. Zwei Merkmale sind für diese gesellschaftliche Formation prägend: die bürgerliche Gesellschaft als Klassengesellschaft (Marx) sowie die Polarität von Öffentlichkeit und Privatheit (Habermas). Dementsprechend sind soziale Ungleichheit und die Polarität einer öffentlichen und einer privaten Sphäre zentrale Kenn-

6 Engels 1959 [1845].

7 Bahrdt 1998.

8 Krämer-Badoni 1992, S. 27.

Leviathan, 41. Jg., 2/2013 
zeichen der europäischen Stadt. Beide prägen ihre sozialräumliche Struktur, und mit beiden hat sich die deutsche Stadtforschung ausführlich beschäftigt.

»[E]s gibt Wissenschaften, denen ewige Jugendlichkeit beschieden ist, und das sind alle historischen Disziplinen, alle die, denen der ewig fortschreitende Strom der Kultur stets neue Problemstellungen zuführt. Bei ihnen liegt die Vergänglichkeit aller [sic], aber zugleich die Unvermeidlichkeit immer neuer [sic] idealtypischer Konstruktionen im Wesen der Aufgabe. «9 Soziologische Begriffe sind grundsätzlich nur als historische möglich und deshalb nur von begrenzter Haltbarkeit. Die Arbeit am soziologischen Begriff ist der nie abschließbare Versuch, die historische Bewegung des Gegenstands nachzuzeichnen. Deshalb muss die Frage nach der Konstitution des Gegenstands der Stadtforschung auch immer wieder gestellt werden. Aber die Antwort kann kein ahistorischer Begriff von Stadt sein. Wenn Berking ${ }^{10}$ dagegen »Stadt als raumstrukturelle Form der Verdichtung « definiert, versucht er eben das. Ich habe Zweifel, ob ein solcher Begriff von Stadt in seiner gewollten Inhaltsleere für empirische Forschung instruktiv sein kann.

Mit dem Darmstädter Ansatz wird in direktem Anschluss an die drei Kategorien der Chicagoer Schule - Dichte, Größe und Heterogenität - versucht, Stadt als eine ahistorische Kategorie jenseits einer bestimmten gesellschaftlichen Formation zu bestimmen. Dagegen insistiert die aktuelle soziologische Stadtforschung in expliziter Distanz zur Chicagoer Schule auf einem inhaltlich gefassten und deshalb historisch spezifischen Stadtbegriff. Darin liegt der erste Unterschied.

Die zweite Kritik besagt, die Stadtsoziologie habe bisher nur in der Stadt geforscht, nicht $\ddot{u} b e r$ die Stadt. Auch diese Kritik trifft insoweit zu, als die soziologische Stadtforschung zum Beispiel an Erscheinungsformen sozialer Ungleichheit in Städten interessiert ist. Aber wenn die Veränderungen der sozialräumlichen Strukturen, die Prozesse der Suburbanisierung, die Privatisierung des öffentlichen Raums oder der Wandel der Stadtpolitik von der Stadterweiterung über die Flächensanierung bis zur sozialen Stadt und dem behutsamen Stadtumbau untersucht werden, dann greifen solche Arbeiten zentrale Themen der Stadtentwicklung und der Stadtpolitik des 20. und 21. Jahrhunderts auf, allerdings nur insoweit sie für viele Städte typisch sind. Dementsprechend müssen die politischen, ökonomischen und sozialen Erklärungen für diese Veränderungen oberhalb der Ebene einer einzelnen Stadt gesucht werden. Der Ansatz der Eigenlogik dagegen ist vorrangig an solchen Gründen interessiert, die einer spezifischen Stadt eigentümlich sind. Darin liegt der zweite Unterschied.

Drittens wird kritisiert, die Stadtsoziologie habe die Differenzierungen des Städtischen vernachlässigt. Dieser Punkt der Kritik ist auf den ersten Blick schwer nachvollziehbar. Lange bevor die Differenzierung zwischen wachsenden und schrumpfenden Städten im West-Ost-Gefälle unübersehbar geworden war und lange bevor diese Differenz in anderen Disziplinen oder der Stadtpolitik thematisiert worden ist, wurde in der Stadtsoziologie auf den grundlegenden Bruch im bislang einheitlichen

9 Weber, zitiert nach Steinert 2010, S. 187.

10 Berking 2008, S. 29. 
Modus der Stadtentwicklung hingewiesen ${ }^{11}$ und damit auf eine heute die Raumordnungs-, Regional- und Stadtpolitik wie auch einen Großteil der raumbezogenen Forschung beherrschende Differenz zwischen Städten. Dass diese unterschiedlichen Entwicklungstypen ihrerseits wiederum von erheblicher Bedeutung für lokale Differenzierungen allgemeiner gesellschaftlicher Entwicklungen sind, haben Goebel et al. ${ }^{12}$ kürzlich bezüglich der Einkommenspolarisierung nachgewiesen. Danach wachsen seit der Jahrtausendwende die Ränder der Einkommensverteilung, das heißt die Zahl der Bezieher von niedrigen Einkommen und die Zahl der Einkommensreichen nehmen zu. Gleichzeitig entwickeln sich die Einkommens-Mittelwerte beider Gruppen in entgegengesetzte Richtungen: Die Armen werden ärmer, die Reichen reicher. ${ }^{13}$ Diese gesellschaftliche Entwicklung wird jedoch lokal je nach Typus der Stadt unterschiedlich gefiltert. In allen von ihnen untersuchten vier städtischen Entwicklungstypen schrumpft die Mittelschicht. Aber die Einkommenspolarisierung ist in den prosperierenden und hier besonders in den rein tertiär geprägten Stadtregionen sehr viel ausgeprägter als in jenen, deren Entwicklung vom Schrumpfen dominiert ist. ${ }^{14}$

Die funktionalen und sozialräumlichen Differenzierungen innerhalb von Städten, die Mischung und Entmischung der städtischen Funktionen und insbesondere die soziale Segregation sind geradezu das zentrale Thema der Stadtsoziologie seit den Anfängen empirischer Stadtforschung in Chicago. Dabei geht es nicht nur um ökonomische, sondern ebenso um demographische, ethnische und kulturelle Differenzierungen (Alter, Einwanderermilieus, Lebensstile). Einen Mangel an Studien zur Differenzierung zwischen und innerhalb von Städten bis hin zu Tendenzen der Spaltung und Ausgrenzung kann man der bisherigen Stadtsoziologie also kaum vorhalten. Allerdings zielt die soziologische Stadtforschung auch hier auf systematische, verallgemeinerbare Differenzierungen und auf Typenbildung. Der Darmstädter Ansatz dagegen konzentriert sich innerhalb der Städte wie zwischen diesen auf Differenzen vorwiegend kultureller Art. Und es geht dabei nicht darum, Städte anhand eines tertium comparationis vergleichbar zu machen. ${ }^{15}$ Der Vergleich soll vielmehr dazu dienen, gerade die Unvergleichbarkeit, die Singularität der einzelnen Stadt herauszuarbeiten. Der Darmstädter Ansatz verfolgt - so scheint es - ein ideographisches Interesse im Gegensatz zum nomothetischen Interesse in der sonstigen soziologischen Stadtforschung. Darin liegt der dritte Unterschied.

\section{Neugründung der Stadtsoziologie?}

Wenn die Unterscheidung von nomothetischem und ideographischem Erkenntnisinteresse die beiden Auffassungen von Stadtsoziologie richtig charakterisieren sollte,

11 Häußermann, Siebel 1986.

12 Goebel et al. 2012.

13 Ebd., 385.

14 Ebd., S. 389, Abbildung 5.

15 Löw 2008, S. 48.

Leviathan, 41. Jg., 2/2013 
dann läge darin ein entscheidender Unterschied sowohl im Bezug auf Politik wie in der grundsätzlichen Auffassung von Soziologie. Im Pathos, mit dem der EigenlogikAnsatz zuweilen vorgetragen wird, schwingt - so scheint es - neben dem Respekt für die Einzigartigkeit jeder Stadt auch eine sehr grundsätzliche politische Kritik mit, die einer nomothetischen Wissenschaft vorhält, Steuerungswissen zu produzieren und damit als Agent von Herrschaft zu fungieren. Diese Kritik wäre in dieser Allgemeinheit auch berechtigt. Ein solcher Vorwurf müsste allerdings auch den Darmstädter Ansatz treffen, der selber keineswegs frei ist von Bezügen auf praktische Politik. In ihrem Buch Soziologie der Städte ${ }^{16}$ begründet Martina Löw den Ansatz der Eigenlogik unter anderem auch damit, nur in Kenntnis ihrer Eigenlogik könne eine Stadt effektiv gesteuert werden. Die Nähe einiger im Rahmen dieses Ansatzes behandelter Themen zum Stadtmarketing scheint offenkundig, ${ }^{17}$ und ein Buchtitel wie »Typisch Darmstadt «18 taugte auch als Slogan für eine Kampagne der Stadtwerbung.

Aber das Pathos hat noch einen zweiten und wichtigeren Grund. Mit dem Ansatz der Eigenlogik scheint mehr beabsichtigt zu sein als nur eine Ergänzung und Vertiefung der soziologischen Stadtforschung um bisher vernachlässigte Themen. Beansprucht wird nicht weniger als eine völlige Neuorientierung der Stadtsoziologie. Die Notwendigkeit paradigmatischer Veränderungen in der soziologischen Forschung ließe sich auf zweierlei Weise begründen. Einmal mit einem Wandel der Theorie, der die Wissenschaft befähigt, eine vorgegebene Wirklichkeit adäquater zu erkennen (1). Zum anderen mit einem Wandel der Realität, durch den sich die Stadtsoziologie in ihren Strukturen so weitgehend ändert, dass darauf mit einer grundsätzlichen Erneuerung der Theorie geantwortet werden muss (2). Aber nicht immer ist der Forscher so viel schlauer geworden, dass er mit neuen Begriffen die vorher undurchdrungene Wirklichkeit nun in aller Klarheit erfassen könnte, und sozialer Wandel ist allenfalls nach einem Zeitenbruch, also, wie anfangs angesprochen, auf lange Frist (longue durée) so radikal, dass sich neue Begriffe dafür aufdrängen würden.

\section{(1) Bessere Theorie}

Was eine Theorie in den Blick nehmen kann und wovon sie absehen muss, das macht ihre Qualität aus. Wenn eine Theorie unscharf oder widersprüchlich ist oder wesentliche Aspekte ihres Gegenstandsbereichs systematisch ausblendet, dann kann und muss sie durch eine bessere Theorie abgelöst werden. Dieses Argument scheint mir im Darmstädter Ansatz zentral zu sein. Die bisherige Forschung habe die Besonderheit des Ortes und die Eigenlogik des Städtischen nicht wahrgenommen. Jenseits der Differenz zwischen Typen von Städten und über das Interesse am besonderen Fall als einer Abweichung von der Regel hinaus sei es Aufgabe der Stadtsoziologie, die Singularität jeder Stadt zu beschreiben. Aber die Vernachlässigung des Besonderen jeder einzelnen Stadt beruht auf theoretischen Vorentscheidungen, die mit der

16 Löw 2010, S. 18.

17 Vgl. ebd., Kapitel V.

18 Löw et al. 2010. 
grundsätzlichen Auffassung von Soziologie zu tun haben. Jede wissenschaftliche Disziplin thematisiert nur einen kleinen Ausschnitt aus der Wirklichkeit. Soziologie ist nicht am Individuum interessiert, sondern an dem, was am Individuum als Gesellschaftliches sichtbar wird. Soziologie, das ist die Kränkung, die sie dem Alltagsbewusstsein zufügt, insistiert darauf, dass noch in den scheinbar individuellsten Entscheidungen (Selbstmord, Partnerwahl oder Musikgeschmack) Gesellschaftliches wirksam ist. Eben darauf zielt auch der Bourdieu'sche Habitusbegriff, ${ }^{19}$ und jede sozialwissenschaftliche Statistik wiederholt diese Kränkung. Deshalb hat sich die Stadtsoziologie eben nicht für die Singularität einer Stadt interessiert, sondern für das, was an Städten und ihren Entwicklungen und auch am einzelnen Fall einer bestimmten Stadt verallgemeinerbar ist.

Die Soziologie ist als Reflexion auf die tiefgreifenden gesellschaftlichen Veränderungen im 18. und vor allem 19. Jahrhundert entstanden. Das gilt ebenso für ihre Teildisziplinen: Die Industriesoziologie untersucht den Wandel von Organisation und Bedingungen der Arbeit im Zuge der kapitalistisch organisierten Industrialisierung, die Familiensoziologie den Wandel von Funktion und Struktur der Familie im Zuge des demographischen Wandels und der Auslagerung produktiver Arbeit aus dem Haushalt und die Stadtsoziologie den Wandel von Siedlungsstruktur, Lebensweise und Mentalität im Zuge der Urbanisierung. Dabei geht es jeweils um allgemeine, überindividuelle Veränderungen. Comte und Durkheim haben die Soziologie begründet als eine Wissenschaft, die sich mit dem Ausschnitt der sozialen Wirklichkeit befasst, der, um mit Marx zu sprechen, über die Köpfe hinweg und durch die Köpfe hindurch sich herstellt. Der Ansatz der Eigenlogik und damit die Frage nach der Singularität jeder einzelnen Stadt wird von Berking und Löw nun nicht aus der Theorietradition der Soziologie begründet, sondern aus den Selbstverständlichkeiten alltäglicher Erfahrung. In ihren Texten finden sich ausdrucksvolle Zitate aus der schönen Literatur und mit Formulierungen wie »steht außer Frage ", "zweifellos ", »ist schwer zu bestreiten « etc. die Berufung auf ein selbstverständliches Alltagswissen von der Einzigartigkeit jeder Stadt. Eben von dieser unbestreitbaren Tatsache habe Stadtsoziologie auszugehen. Und in der Tat »ist schwer zu bestreiten ", dass Wanne-Eickel nicht New York und Düsseldorf nicht Köln ist. Ebenso wenig wäre zu bestreiten, dass VW nicht Siemens ist und die Familie X eine ganz andere Familie als die Familie Y. Aber trotzdem konstituiert sich die Industriesoziologie nicht durch die Singularität von VW und die Familiensoziologie nicht durch die Einmaligkeit der Familie X. Wenn einzelne Fälle untersucht werden, dann nicht, um ihre Einzigartigkeit zu erfassen, sondern um auszuloten, wie viel Spielraum für die Interpretation von familialen Rollen besteht oder wie weit die Spannbreite möglicher Unternehmenskulturen angesichts eines global gewordenen Wettbewerbs ist Fragen also, die auf die Rigidität beziehungsweise Offenheit gesellschaftlicher Strukturen zielen. Ähnlich sucht die Politikforschung mittels vergleichender Fallstudien die Handlungsspielräume und eigenständigen Ressourcen lokaler Politik angesichts globaler Standortkonkurrenz und der Vorgaben überlokaler politischer Ebenen zu bestimmen.

19 Gestring 2011, S. 45 f.

Leviathan, 41. Jg., 2/2013 
Städte sind unter bestimmten Blickwinkeln kaum vergleichbare Individualitäten. Das gilt für ihre Topographie, ihre Architektur, ihren Städtebau, ihre Geschichte. Und deshalb sind diese Individualitäten auch von Architekten, Städtebauern, Geographen und Historikern gut zu beschreiben und im Bezugssystem ihrer Wissenschaften zu analysieren. Aber die Soziologie ist nicht an Einzigartigkeiten interessiert. Ein Verzicht auf den Begriff der Gesellschaft, wie ihn Helmuth Berking fordert, wäre der Verzicht auf Soziologie. Unabhängig davon, ob von lokaler, nationaler oder Weltgesellschaft die Rede ist, es ist die Orientierung ihres Erkenntnisinteresses auf Gesellschaft, die die Soziologie von anderen Wissenschaften wie Historie, Ethnologie oder Psychologie unterscheidet. Darin liegen auch die Grenzen der Soziologie. Aber wenn Soziologen darauf verzichten, arbeiten sie als schlecht ausgebildete Historiker, Ethnologen oder Psychologen. Gerade interdisziplinäre Kooperation setzt bei den beteiligten Disziplinen ein Bewusstsein ihrer spezifischen Leistungsfähigkeit und ihrer spezifischen Begrenzungen voraus.

\section{(2) Sozialer Wandel}

Die zweite Rechtfertigung einer Neuorientierung (oder »eines Paradigmenwechsels«) der Stadtsoziologie auf Basis der Kategorie Eigenlogik müsste mit einem Wandel des Gegenstands Stadt argumentieren, der so tiefgreifend ist, dass die überkommenen Fragestellungen, Erklärungen, Begriffe und Methoden der Stadtforschung ihre Gültigkeit verlieren. Gibt es im Gegenstandsbereich der Stadtforschung Entwicklungen, die die Eigenlogik der Städte so unabweisbar in den Fokus der Stadtforschung drängen, dass eine neue, auf dieser Kategorie basierende Stadtsoziologie notwendig geworden wäre?

Mit der Kategorie Eigenlogik wird behauptet, dass »Bebauungsformen und Architekturen, $[\ldots]$ geologische und klimatische Differenzen oder $[\ldots]$ materialisierte soziale Figurationen ${ }^{20}$ »Strukturmuster « erzeugen, »die als `Ortseffekte die Handlungen aller sozialer Gruppen durchziehen «, ${ }^{21}$ sodass sie »quer zu den Milieus Taxifahrer/-innen, Hochschullehrer/-innen, Tänzer/-innen und Priester et cetera einer [sic] Stadt gemeinsame Praxisformen ausprägen $« .{ }^{22}$ "Widersprüche und milieuspezifische Differenzen sind möglich, bleiben aber auf die Struktur der Stadt insbesondere durch dominante Deutungsmuster bezogen. «23 Bei derart starken Formulierungen der Eigenlogik-These als »dominanter "Struktur wünscht man sich eine Auseinandersetzung mit der reichlich vorhandenen Literatur zu städtischen Milieus und Subkulturen, die eher das Gegenteil nahelegen. Die Milieu-Studien der Chicagoer Schule hatten schon Robert Park dazu geführt, Stadt nicht als »Erfahrungsgemeinschaft « ${ }^{24} \mathrm{zu}$ konzipieren, sondern als ein Mosaik kleiner Welten mit einer jeweils eigenen Mentalität (moral order).

20 Löw 2008, S. 44.

21 Ebd., S. 40.

22 Ebd., S. 45.

23 Ebd., S. 50.

24 Löw 2010, S. 62. 
Löw und Noller benennen fünf Strukturen, die im Rahmen von Forschungen zur Eigenlogik einer Stadt untersucht werden sollen: » Raumstrukturen, Zeitstrukturen, Sozialstrukturen, Politische Strukturen, Gefühlsstrukturen «. ${ }^{25}$ Sicherlich gibt es in allen diesen Dimensionen Differenzen zwischen Städten. Aber Bremer Großbürger oder Bremer Arbeitslose werden hinsichtlich alltäglicher Lebensorganisation, Mentalität, politischer Orientierung, Lebenslage etc. mehr Ähnlichkeiten mit Essener Großbürgern respektive Arbeitslosen aufweisen als unter sich. Kurz: Sozialstrukturelle und Lebensstilfaktoren sind im Kontext soziologischer Fragestellungen sehr viel erklärungskräftiger als die Besonderheiten einer Stadt. Wenn bei sonst gleichen strukturellen Bedingungen unterschiedliche städtische Politiken beobachtet werden, dann können Färbungen lokaler Arenen (Blanke) oder regionale Steuerungsstile (Fürst) dafür tragfähige Erklärungen anbieten. Aber durch die Suche nach lokalspezifischen Faktoren der Erklärung werden die überlokalen strukturellen und institutionellen Rahmenbedingungen nicht entwertet. Entscheidend ist, von welcher Relevanz für die Erklärung des je Vorgefundenen die beobachtbaren lokal spezifischen Variationen sind.

Die Themen, an denen die Relevanz eigenlogischer Strukturen in den vorliegenden Texten illustriert wird, klammern klassische Fragen soziologischer Forschung weitgehend aus. Sie fügen sich in den cultural turn: Gefühle, Einstellungen, alltägliche Verhaltensweisen, Wahrnehmungsmuster ... Nicht dass solche Themen gänzlich irrelevant wären, aber was hat man über die Sozialstruktur der Bundesrepublik gelernt, wenn man festgestellt hat, dass sich Armut in verschiedenen Städten verschieden » anfühlt «, ${ }^{26}$ und was weiß man als Soziologe, wenn man erfahren hat, dass die Schrittgeschwindigkeit in Berlin höher ist als in München oder Darmstadt? ${ }^{27}$ Ein empirischer Beleg, der zur Begründung des Darmstädter Ansatzes angeführt wird, ist die Studie von Taylor et al., ${ }^{28}$ in der die unterschiedlichen Weisen, mit denen Manchester und Sheffield auf die Deindustrialisierung reagiert haben, auf unterschiedliche Mentalitäten beider Städte zurückgeführt werden: in Manchester eine Kultur des Wandels, in Sheffield Trauer um die vergangene Größe. Dass diese Unterschiede statt einer Ursache die Folge unterschiedlicher Entwicklungspfade sein könnten, taucht in den Überlegungen gar nicht auf.

Es war eine zentrale These der Gemeindesoziologie, dass die Stadt eine eigenständige Form der Vergesellschaftung sei. Für René König war »die Gemeinde neben der Familie eine der wichtigsten Grundformen der Gesellschaft « ${ }^{29}$ und eine »Wirklichkeit eigener Natur « ${ }^{30}$, eine Instanz der Sozialisation von erheblicher Bedeutung für die Gemeindemitglieder. Croon und Utermann ${ }^{31}$ haben das zum Thema

25 Löw, Noller 2010, S. 259.

26 Löw 2010, S. 18.

27 Löw, Noller 2010, S. 260.

28 Taylor et al. 1996.

29 König 1958, S. 7 f.

30 Ebd., S. 8.

31 Croon, Utermann 1958.

Leviathan, 41. Jg., 2/2013 
gemacht. Sie konnten zeigen, wie die Tatsache, in einer Zechengemeinde aufzuwachsen, die Lebensziele von Jugendlichen einengen kann auf jene Perspektiven, die der Bergbau anbietet. Hans Oswald hat die Ergebnisse dieser Studien schon vor 47 Jahren auf den Nenner überlokaler Determination und lokaler Filterwirkung gebracht. Er resümiert seine Untersuchung zur Reichweite von Gemeindestudien: "[...] daß es zwar gewisse Gemeinsamkeiten gibt, daß das gemeindliche Determinationssystem einen geringen Einfluß ausübt, daß es Überreste von Ortsbezogenheit in bestimmten Bevölkerungskreisen gibt, daß die meisten Bewohner und Besucher einer Stadt einen Eindruck von ihrer Eigenart, ihrem Charakter und ihrer Atmosphäre haben. Andererseits ist dies alles von sekundärer Bedeutung. Die entscheidenden Determinanten sozialen Lebens sind heute nicht mehr gemeindlicher Natur. Die Menschen leben zwar zum größten Teil in Gemeinden, aber ihr gemeindliches [sic] Zusammenleben kann nicht mehr der Ansatz zu ihrer Erforschung sein «. ${ }^{32}$ Das liest sich wie eine Kritik am Darmstädter Eigenlogik-Ansatz. Aber in den Texten zur Eigenlogik gibt es nirgends eine Auseinandersetzung mit den doch sehr verwandten Traditionen der Gemeindesoziologie, schon gar nicht mit der Kritik daran, ebenso wenig mit den Theorien, die heute auf einen weiteren Bedeutungsverlust der lokalen Ebene für sozialwissenschaftliche Fragestellungen hinweisen könnten.

Von Marx und Weber über Simmel bis zur Systemtheorie bieten die Theorien der modernen Gesellschaft Argumente, die für eine Entwicklung in Richtung auf Entwertung des Lokalen sprechen. ${ }^{33} \mathrm{Im}$ Kommunistischen Manifest prognostizieren Marx und Engels, ${ }^{34}$ dass der Kapitalismus den Markt räumlich wie inhaltlich immer weiter ausdehnt bis zur weltumspannenden Organisation einer globalen Ökonomie. Simmel ${ }^{35}$ hat die ungeheure Abstraktionskraft des Geldes als eines ortlosen Mediums sozialer Beziehungen analysiert. Es liege in der Logik der Geldwirtschaft, einen Weltmarkt zu schaffen, der die Menschen aus ihren räumlichen Verbundenheiten löst. Norbert Elias’ Annahme sich immer stärker ausweitender Interdependenzket$\operatorname{ten}^{36}$ oder Stichwehs »Weltgesellschaft « ${ }^{37}$ verweisen ähnlich wie Simmels Geldtheorie darauf, dass soziale Zusammenhänge immer mehr von immer ausgreifenderen räumlichen Bezügen bestimmt werden. Wissenschaftliche Disziplinen, Märkte, Sprachgemeinschaften und religiöse Konfessionen bilden zugleich eigene Räume dichter Kommunikation. Nur in Sonderfällen sind diese identisch mit physischen Räumen. ${ }^{38}$

Dementsprechend lassen sich empirische Tendenzen nennen, die auf einen Abbau lokaler Eigenart hindeuten, insbesondere der Wandel in der Produktion von Stadt:

32 Oswald 1966, S. 192.

33 Das Folgende nach Garhammer 2000.

34 Marx, Engels 1980 [1848], S. 465 f.

35 Simmel 1989.

36 Elias 1976.

37 Stichweh 2000, S. 202.

38 Nassehi 2002, S. 220. 
Mönninger ${ }^{39}$ und Grubbauer ${ }^{40}$ beschreiben den wachsenden Einfluss von an Lokalität nicht interessierten Akteuren auf Stadtentwicklung und Architektur. Der bürgerliche Bauherr, der auf seinem Grundstück mit seinem Architekten für seine Familie oder sein Geschäft ein Haus errichtet, ist zur Ausnahme geworden. Seine Rolle wird arbeitsteilig aufgespalten auf Investoren, Entwickler, Bauträger, Architekten und Ingenieure. Das fertige Gebäude wird von einem Immobilienfonds gekauft, der es an vorher nicht bekannte Nutzer vermietet. Das erzwingt eine möglichst neutrale Architektur, die offen ist für die unterschiedlichsten Nutzungen. Für sogenannte Flagship-Architekturen werden Großarchitekten engagiert, deren Namen für internationale Aufmerksamkeit sorgen sollen, nicht für eine vorsichtige Weiterentwicklung des genius loci. Ebenso wie der bürgerliche Bauherr ist auch das inhabergeführte Unternehmen in den Städten auf dem Rückzug. Die Filialisierung der Innenstädte führt zu einer Standardisierung von Warenangebot und Erscheinungsform. Auch die nationale und die europäische Politik tragen zur Angleichung lokaler Differenzen bei. Das Grundgesetz verpflichtet die Raumordnungs- und Regionalpolitik, gleichwertige Lebensverhältnisse in allen Teilen der Bundesrepublik herzustellen. Seit dem Städtebauförderungsgesetz versucht der Bund, die Städte in den Dienst zentralstaatlicher Wachstums- und Konjunktursteuerung zu nehmen. ${ }^{41}$ Die im Kielwasser eines dominant gewordenen Neoliberalismus vorgenommenen Privatisierungen kommunaler Dienstleistungen haben die Handlungsspielräume kommunaler Politik ebenso geschwächt wie die strukturelle Finanzmisere der Kommunen. Die Internationalisierung des deutschen Wohnungsmarkts nimmt den Kommunen ihre wichtigsten Partner einer lokal verantwortlichen Stadtpolitik, die gemeinnützigen Wohnungsbauträger. An ihre Stelle treten international tätige Investoren, die häufig keinerlei Interesse zeigen, sich an lokaler Politik zu beteiligen. ${ }^{42}$ Schließlich richtet sich die Stadtpolitik neuerdings auf eine Zielgruppe von hochqualifizierten Managern, Ingenieuren und sogenannten Kreativen. Die Angehörigen dieser Arbeitskraftelite sind auf internationale Arbeitsmärkte orientiert. Die Stadt, in der sie sich jeweils für eine begrenzte Zeit ihrer Karriere aufhalten, hat für sie eher Hotelfunktion, und sie verlangen Dienstleistungen und Infrastrukturen, die internationalen Standards genügen, also zum Beispiel gerade keine kultur- oder gar lokalspezifischen Bildungseinrichtungen, sondern internationale Schulen, die an jedem Ort der Welt ein möglichst ähnliches Curriculum bieten.

Beispiele lassen sich immer durch Gegenbeispiele entkräften, und bei dem hier anstehenden Thema können diese sich ebenfalls auf theoretische Argumente stützen. Die Wissensökonomie weist eine hohe Affinität zu urbanen Standorten auf, und die Einbettung in ein dichtes Geflecht lokaler Kooperationen dient als Rückversicherung angesichts der enormen Unsicherheiten in einer globalisierten Wirtschaft. "Städte sind [...] flexible Versicherungen [...], denn ihre Umgebung und ihre Ver-

39 Mönninger 2012.

40 Grubbauer 2012.

41 Siebel 1974.

42 BBSR 2011, S. 4.

Leviathan, 41. Jg., 2/2013 
schiedenheit machen es Firmen möglich, ihre Risiken zu reduzieren und Zugang zu großen Ressourcen an Fachwissen, Finanzierung und Infrastruktur auf dem Arbeitskräftemarkt zu finden, wodurch ihre Unsicherheiten angesichts der Zufälle des globalen Wettbewerbs eingegrenzt werden. ${ }^{43}{ }^{43}$ Läpple $^{44}$ spricht mit Bezug auf diese Argumente von einer »Neuerfindung der Stadt «. Auch gibt es Hinweise auf eine politisch vorangetriebene »Re-Kommunalisierung`von öffentlichen Dienstleistungen $«{ }^{45}$ Aber ob es sich dabei um dominante Trends handelt oder ob die Tendenzen, die das Lokale wieder in Wert setzen, eher den Charakter reaktiver Kompensationen haben, ist umstritten. »Politische oder kulturelle Traditionen sind Objekte der Wiedererfindung « ${ }^{46}$ denn der Vormarsch von Marktkräften und -ideologien »hat in manchen Bereichen und in unterschiedlicher politischer Art und Weise einen Bedarf an politischer und sozialer Organisation erzeugt, der nicht auf der nationalen Ebene angesiedelt ist. Die Ausbreitung des Marktes scheint paradoxerweise zu einer Rückkehr binnenstaatlicher politischer Einheiten wie der Städte zu führen [...] mit dem doppelten Ziel des Versuchs, an den ökonomischen Wandel Anschluss zu finden und zugleich die Wirkungen des Marktes in gewisser Weise auszugleichen «. ${ }^{47} \mathrm{Löw}$ legt eine solche kompensatorische Erklärung der Aktualität von Eigenlogik selber nahe: Forschungen zur Eigenlogik der Städte seien auch deshalb notwendig, um der Gefährdung der besonderen Identität von Städten durch die Homogenisierung ihres Erscheinungsbilds im Zuge der Globalisierung zu begegnen. ${ }^{48}$

Der Anspruch von Berking und Löw, die Stadtsoziologie neu zu gründen, ist überzogen. Er wird von den Autoren weder theoretisch noch empirisch überzeugend begründet. Das Pathos, mit dem er zuweilen vorgetragen wird, speist sich nicht zuletzt daraus, dass die vorliegende soziologische Stadtforschung nur sehr selektiv wahrgenommen wird. ${ }^{49}$ Der Darmstädter Ansatz liefert kein neues Paradigma für die Stadtsoziologie, er ergänzt freilich die bisherige soziologische Stadtforschung, indem Forschungsfelder benannt, Fragen gestellt und lokalspezifische Erklärungen betont werden, die in der Stadtforschung bislang nicht oder - mit guten Gründen -

$43 \gg$ Cities are [...] flexibility insurances [...], because their environment and their diversity enable firms to cut their risks and to have access to huge ressources in expertise, finance and infrastructure on the labour market which reduce their margin of uncertainty in the face of the hazards of global economic competition. " (Bagnasco, Le Galès 2000, S. 20; Übersetzung W.S.)

44 Läpple 2005.

45 Wollmann 2013.

46 » Political or cultural traditions are subject to reinvention, [...].« (Bagnasco, Le Galès 2000, S. 25; Übersetzung W.S.)

$47 »[\ldots]$ has in some areas and in different political forms produced the demand for a level of political and social organisation other than national. The spread of the market appears paradoxically to be leading to a return of the political infrastate territories such as cities [...] with the twofold aim of attempting to adapt to economic change and of counterbalancing somewhat the effects of the market. " (Ebd.; Übersetzung W.S.)

48 Löw 2010, S. 122 f.

49 Häußermann 2011; Schäfers 2011. 
nicht mehr im Mittelpunkt gestanden haben. Der Ansatz der Eigenlogik richtet sich im Sinne des cultural turn auf Themen wie die Rolle der Subjekte, die Materialität des Städtischen, auf die sinnlichen Erfahrungen von Stadt, auf Gefühle, Körper und Körperbewusstsein. Aber dadurch werden die Fragen nach den Zusammenhängen zwischen städtischem und gesellschaftlichem Wandel und nach verallgemeinerbaren Prozessen der Stadtentwicklung nicht obsolet. Erst recht lässt der Ansatz der Eigenlogik keine alternative Theorie erkennen, die die vorliegenden Daten zur Stadtentwicklung besser erklären würde als strukturelle Theorien.

\section{Fragen}

Es gibt einige Stellen, an denen das, was mit dem Darmstädter Ansatz erreicht werden soll, bescheidener formuliert ist: »[...] nach Vergesellschaftungsmustern quer zum Milieu zu fragen heißt [...] nicht, gesellschaftliche Struktur (im Singular) in ihrer Potenz und die mit ihr etablierte soziale Ungleichheit und Raumverteilung zu leugnen. Die Frage nach Eigenlogik und Praxis soll aber den Horizont weiten, um Neues an der Stadt und ihren Qualitäten entdecken zu können « ${ }^{50}$ Die bisherige soziologische Stadtforschung sei »nicht ausreichend vielfältig « ${ }^{51}$, weshalb »Überlegungen zu einem möglichen Perspektiv- und Erkenntnisgewinn durch eine ergänzende Hinwendung zum Gegenstand \Stadt $<$ formuliert « 52 werden sollen. Aber auch wenn der überzogene Anspruch auf ein neues Paradigma für die Stadtsoziologie letztlich nicht aufrechterhalten bliebe, würden sich Probleme stellen, die gelöst werden müssen, sollte der Ansatz über die Phase mutiger Hypothesenformulierung hinaus gelangen zu belastbarer empirischer Forschung.

\subsection{Die Frage der soziologischen Relevanz}

Die Grundthese von der Singularität jeder Stadt ist plausibel. Lokale Variationen in der Handhabung von Regulierungen der Prostitution, von Karnevalsfeiern und Demonstrationen am Christopher Street Day, im Verhalten von Taxifahrern und beim Gang zu Haltestellen des ÖPNV, wie sie von Löw als Beispiele genannt werden, mögen die Singularität einer Stadt belegen, aber fügen sie sich in eine Eigenlogik, »die die Stadt wie ein Rückgrat durchzieht «? ${ }^{53}$ Vor allem aber: Welche soziologische Relevanz haben solche Erkenntnisse? Die bisherige Stadtforschung war bemüht, an die klassischen Fragen der Soziologie anzuschließen: Was treibt Gesellschaft voran? Was hält Gesellschaft zusammen? Wie ist die soziale Struktur einer Gesellschaft beschaffen? Die Stadtsoziologie hat zur Erklärung und Beschreibung sozialen Wandels beigetragen, indem sie zum Beispiel Prozesse der Urbanisierung untersucht als Änderung der Siedlungsform (Verstädterung) einerseits und als Urbanisierung im

50 Löw 2008, S. 50.

51 Löw 2010, S. 31.

52 Ebd., S. 25.

53 Löw 2008, S. 37.

Leviathan, 41. Jg., 2/2013 
eigentlichen Sinne andererseits, also als Wandel der Lebensweise durch Vergesellschaftung der Reproduktion, durch Trennung von Wohnen und Arbeiten. Der Prozess der Urbanisierung ist keineswegs abgeschlossen. Die gegenwärtig diskutierte »Renaissance der Städte « ist nicht nur ökonomisch zu erklären, sondern auch durch soziale Veränderungen, insbesondere die Veränderungen der Lebensweise im Zuge des Wandels der Rolle der Frau. ${ }^{54}$ Fragen der gesellschaftlichen Integration spielen eine zentrale Rolle, seit Simmel mit der urbanen Lebensweise einen besonderen Modus städtischer Integration beschrieben hat, der anders als die Integrationsleistung dörflicher Gemeinschaften nicht auf Homogenität setzt, sondern auf das Ertragen von Differenz. ${ }^{55}$ Die Rolle der Stadt als Ort, an dem soziale Ungleichheit in spezifischen räumlichen Formen sichtbar wird (Segregation), und als Ursache für spezifische Formen sozialer Ungleichheit (Ausgrenzung) ist geradezu das klassische Thema der Stadtsoziologie.

Der Darmstädter Ansatz thematisiert stadttypische Gefühlsstrukturen und die unterschiedlichen Muster, mit denen verschiedene Städte beschrieben werden. Mit der Kategorie »Phlegmatisch « charakterisieren Löw und Noller beispielsweise die »bildhafte Verdichtung der affekthaften Zuschreibungen ${ }^{56}$ zu Darmstadt. Aber einmal abgesehen davon, ob "phlegmatisch « wirklich die "Menschen (die in der Stadt wohnen, die sie besuchen, die pendeln) in ihren Praktiken, das heißt in ihrer Identität, in Gefühlen, Einstellungen, im Denken prägt «,${ }^{57}$ so wäre auch gegenüber einer pragmatischeren Forschung zur Eigenlogik der Städte zu fragen, was daran für die Soziologie wichtig ist. Unterstellt, solche Fragen könnten in der Tat das Spezifische von Stadt erfassen, so ist damit noch kein Gegenstand einer Soziologie der Stadt gewonnen: »Es könnte nämlich folgendes Paradoxon auftreten: Je mehr wir etwas spezifisch Städtisches zum zentralen Gegenstand der Stadtsoziologie machen, desto weniger lassen sich auf die solcherart bestimmte Stadtsoziologie allgemeine Theorien der Soziologie anwenden «.58 Solche Fragen nach dem Besonderen der Stadt, wie sie im Rahmen des Eigenlogik-Ansatzes gestellt werden, sind wahrscheinlich für Psychologen interessant, aber solange der Beitrag dieser Forschungen zu den zentralen Fragen der Soziologie im Unklaren gelassen wird, wäre der Vorwurf einer Stadtsoziologie ohne Stadt, den die Darmstädter der bisherigen Stadtsoziologie machen, an sie zurückzugeben: Kann es eine Stadtsoziologie ohne Soziologie geben?

\subsection{Die Frage der Operationalisierung}

Der Darmstädter Ansatz hat ein beeindruckendes Forschungsprogramm formuliert: die städtebauliche Gestalt, die Geschichte, die Relation zu anderen Städten, die ökonomischen, kulturellen, sozialen, politischen und materiell-baulichen Dimensionen städtischer Entwicklungen, die verschiedenen Ressourcen, sozialen Praktiken

54 Siebel 2012, S. 208.

55 Siebel 2010.

56 Löw, Noller 2010, S. 264.

57 Löw et al. 2010, S. 13.

58 Friedrichs 2011, S. 33. 
und Aneignungsprozesse, die institutionellen und symbolischen Ordnungen, die Geräusche, Gerüche, der Geschmack, die optischen Eindrücke, Stimmungen und Träume, die sich mit einer Stadt verbunden haben, all das soll einbezogen werden. Angesichts eines derart heroischen Forschungsprogramms drängt sich die kleinmütige Frage auf, ob ein Stadtbegriff, der beansprucht, "sämtliche Verhältnisse des urbanen Raums in ihrem relationalen Zusammenhang zu begreifen " ${ }^{59}$ für empirische Forschung überhaupt operationalisierbar sein kann. Dass alles mit allem zusammenhängt, ist zwar immer wahr, aber ist es auch ein erfolgversprechender Ansatz für empirische Forschung? Anders gesagt: Benötigt nicht auch der Eigenlogik-Ansatz eine Theorie, die es erlaubt, in der prinzipiell unendlichen Fülle des empirisch Vorfindlichen Relevantes von Irrelevantem zu unterscheiden? Ohne eine theoretisch begründete Selektion dessen, was beobachtet werden soll und was vernachlässigbar ist, gerät empirische Forschung in die Falle der Beliebigkeit oder in einen Regress ins Unendliche.

\subsection{Unklare Begriffe}

Die dritte Frage zielt auf eine konkretere Bestimmung dreier zentraler Kategorien des Darmstädter Ansatzes: Eigenlogik, Stadt und Dichte.

\section{Eigenlogik}

Nach Zimmermann meint »Eigenlogik [...], dass Städte eigene Funktions- und Ordnungsmuster ausprägen $«{ }^{60}$ Gegenstand eigenlogischer Forschungen sind »die Bedeutung symbolisch-sinnhafter Regeln, [...] sowie der Stellenwert kollektiv geteilter Wissensordnungen, Symbolsysteme, Sinnhorizonte, die lokale, nationale und globale sinnhafte Ordnungen und ihre symbolische Organisation der Wirklichkeit regulieren « ${ }^{61}$ Die Eigenlogik einer Stadt wie Darmstadt zu verstehen heißt, »wir wollen nachvollziehen, wie Darmstadt zu einem Sinnzusammenhang wird, der Menschen [...] in ihren Praktiken, das heißt in ihrer Identität, in Gefühlen, Einstellungen, im Denken prägt « ${ }^{62}$ Mit Eigenlogik ist demnach eine zeitlich (a), sozial (b) und biographisch (c) relativ stabile, widerspruchsfreie und sich selbst reproduzierende Identität jeder einzelnen Stadt gemeint.

(a) Zeitliche Stabilität: Rodenstein beschreibt in ihrem Beitrag zum Band »Die Eigenlogik der Städte " als Eigenlogik der Stadt Frankfurt am Main das glatte Gegenteil von Kontinuität, nämlich die wiederholten und radikalen Brüche im Selbstverständnis der Stadt. ${ }^{63}$ Über Jahrhunderte habe ein erbliches Patriziat die Stadt beherrscht. Aber aus dieser Kontinuität erwuchs nach Rodenstein keine Eigen-

59 Bockrath 2008, S. 80.

60 Zimmermann 2008, S. 207.

61 Berking, Löw 2005, S. 12.

62 Löw et al. 2010, S. 13.

63 Rodenstein 2008. 
logik, sondern im Gegenteil aus den Brüchen der Frankfurter Geschichte: zuerst der politische Bruch durch die Mediatisierung Frankfurts in Preußen, dann der ökonomische Bruch mit der Ablösung des Handels als dominanter ökonomischer Funktion zunächst durch die industrielle Produktion, später dann durch die Finanzwirtschaft. Diese Brüche waren flankiert von einem Wandel der Eliten: vom stadtsässigen Handelsbürgertum über ein Rentierspatriziat zum Industriekapital und schließlich zu einem (internationalen) Finanzkapital. Alle diese Brüche zwingen, so Rodenstein, zu einer Ablösung von der eigenen Geschichte. Frankfurts Eigenlogik besteht »im ständigen Wandel des Selbstverständnisses « ${ }^{64}$ - ein Wandel, der obendrein durch äußere Umstände erzwungen wurde. Wie ist das mit der Annahme einer sich selbst reproduzierenden eigenlogischen Entwicklung vereinbar? Kann Eigenlogik sowohl Kontinuität, die sich selbst perpetuiert, wie gerade das Gegenteil, eine Abfolge radikaler Brüche, sein?

(b) Soziale Stabilität: Die Eigenlogik einer Stadt prägt den Habitus und die Doxa "quer zu den Milieus «. ${ }^{65}$ Jede individuelle Stadt evoziert »die ihr eigene snatürliche Einstellung zur Welt«.66 Darunter sind »Strukturmuster « zu verstehen, »die als Ortseffekte die Handlungen aller sozialer Gruppen durchziehen « ${ }^{67}$ In der Stadtsoziologie werden dagegen seit Längerem Veränderungen sozialer Ungleichheit in Richtung auf Spaltung, Ausgrenzung oder Polarisierung diskutiert. ${ }^{68}$ Goebel et al. haben sie kürzlich auch für deutsche Städte nachgewiesen. ${ }^{69}$ Wenn aber Ausgrenzung, Spaltung und Polarisierung zu Phänomenen städtischer Ungleichheit werden, wie kann dann eine Eigenlogik der Städte solche gesellschaftlichen Spaltungen noch überwölben?

Kevin Lynch ${ }^{70}$ hatte in seiner Untersuchung über das Bild der Stadt das Bostoner Westend als gänzlich uninteressantes Gebiet beschrieben. In Herbert Gans berühmter Studie über die Urban Villagers ${ }^{71}$ erscheint eben jenes Stadtviertel als ein äußerst lebendiges urbanes Einwandererquartier. Lynch hatte amerikanische Studenten befragt, die das Quartier gar nicht kannten, Gans die Bewohner des Quartiers. Wenn die Stadt stets auch wahrgenommene Stadt ist, wie kann dann angesichts der sozialen Spaltungen der Stadt eine Eigenlogik ausgebildet werden, die noch die Viertel der Ausgegrenzten durchdringt? Jürgen Hasse spricht geradezu von einer Konkurrenz, ja Feindschaft verschiedener, situativ gebundener Stadtvorstellungen: »In einer [sic] Stadt herrscht ein Kampf zwischen [sic] den Städten. Kontrahenten sind die situativen Städte, die als Folge des unterschiedlichen Lebens der Menschen ent-

64 Ebd., S. 309.

65 Löw 2008, S. 45.

66 Berking 2008, S. 27.

67 Löw 2008, S. 40.

68 Kronauer 2002; Koll-Schretzenmayr et al. 2009.

69 Goebel et al. 2012.

70 Lynch o. J.

71 Gans 1962. 
stehen «. ${ }^{72}$ Wie ist da eine widerspruchsfreie Doxa, ein durchgängiger Habitus überhaupt möglich?

Eigenlogik dürfte für eine sesshafte Mittelschicht vielleicht feststellbar sein, aber kaum für die Pole einer sich spaltenden Gesellschaft. Beide leben am Rand der Stadt: die international tätigen Eliten, die sich in einer Stadt nur vorübergehend aufhalten und nicht mehr von ihr erwarten als von einem gut geführten Hotel, und am Gegenpol die Ausgegrenzten, die von dieser Gesellschaft nichts mehr erwarten und deshalb glauben, ihr auch nichts schuldig zu sein. Und beide werden sich auch subjektiv als nicht dazugehörig definieren. Löw erwähnt diesen Einwand selber und schiebt ihn mit einer Geste recht großzügiger Wissenschaftlichkeit beiseite: »Ohne dies infrage stellen zu können oder zu wollen, so soll hier die Hypothese vertreten werden, dass milieuspezifische [...] Praxisformen [...] die Prägung durch eine städtische Struktur durchzieht «. ${ }^{73}$

(c) Biographische Stabilität: Wenn der Ansatz der Eigenlogik prägende Sozialisationsleistungen des Ortes annimmt, muss er eine gewisse Sesshaftigkeit und eine Organisation des Lebens vorwiegend innerhalb einer Stadt unterstellen. Sonst wäre schwer erklärbar, wie eine Stadt den Habitus ihrer Bewohner quer zu allen Milieus prägen soll. "Vergesellschaftung im postmodernen, globalen Zeitalter ist jedoch gerade durch die Verknüpfung vieler geographischer Orte und die Vernetzung in Stadt übergreifenden Zusammenhängen charakterisiert. « ${ }^{74}$ Schon Oswald hatte gegen die Annahme einer prägenden Sozialisationswirkung von Städten mit der hohen Fluktuation der Stadtbevölkerung argumentiert: »Wieviele dieser Menschen, die erst seit einem, zwei, drei oder fünf Jahren in einer Stadt leben, fühlen sich mit dieser Stadt verbunden, bzw. auf welche Weise sind sie verbunden? Sicherlich, sie arbeiten hier, aber die moderne Arbeit ist in der Regel wenig ortsbezogen, sie gehen hier ins Kino, aber die Filme kommen von außerhalb, sie leben hier, aber sie ändern ihre Verhaltensweisen, Moralbegriffe, Einstellungen und Bezogenheiten kaum, wenn sie in eine neue Stadt ziehen $" .{ }^{75}$ Zur Fluktuation der Stadtbevölkerung tritt die zunehmend regionale Organisation des Alltags über verschiedene Gemeinden hinweg: Man wohnt in A, arbeitet in B, kauft ein in C und fährt durch D mit dem PKW, weshalb man mit diesen Gemeinden jeweils sehr selektive Erfahrungen und hoch spezialisierte Erwartungen verbindet. Die Stadt ist nicht mehr die Einheit des Alltags der Menschen. Diese Diffusion des Ortsbezugs wird noch deutlicher beim Phänomen des multilokalen Wohnens bis hin zum transnationalen Wohnen, seien es deutsche Rentner, die ihre Winter auf Mallorca und ihre Sommer in Deutschland verbringen, seien es transnationale Migranten, die sich phasenweise hier und in ihrem Herkunftsland aufhalten. Hohe Mobilität in immer größeren Räumen und eine weltweite Vernetzung sind Kennzeichen der Moderne. Manderscheid sieht in Sesshaftigkeit und geringer Mobilität sogar »tendenziell [...] Ursache und Ausdruck

72 Hasse 2008, S. 332.

73 Löw 2008, S. 44.

74 Manderscheid 2007, S. 55.

75 Oswald 1966, S. 93. 
von Desintegration und Ausschluss «. ${ }^{76}$ Um eine Frage abzuwandeln, die Häußermann in seiner Rezension des Buchs von Martina Löw gestellt hat: Wie lange braucht es, bis man die Doxa verinnerlicht hat, und wie lange braucht man, um sie wieder loszuwerden? Anders gefragt: Wann wurde aus meinem Freund und Kollegen Häußermann ein Tübinger, ein Berliner, ein Kasselaner, ein Bremer und dann wieder ein Berliner, oder ist er immer Waiblinger geblieben? Oder aber war vielleicht der gemeinsame oberitalienische Ort, an dem wir 30 Jahre lang unsere Bücher geschrieben haben, der prägendste von allen?

Also: Wie ist zeitliche, soziale und biographische Stabilität des Habitus einer Stadt, wie sie die Annahme einer Eigenlogik von Stadt unterstellt, denkbar angesichts der Brüche der Stadtgeschichte, der sozialen Spaltungen der Stadt und hoher Mobilität der Stadtbevölkerung? Ist das Konzept Eigenlogik vielleicht an einer Vorstellung von Stadt und Stadtbürgerschaft orientiert, wie sie für das Lübeck des Senators Buddenbrook noch Gültigkeit besessen haben mag, aber nicht für Berlin und Frankfurt heutzutage?

\section{Stadt}

In den Texten von Berking und Löw bleibt unklar, wie eine Stadt, deren Eigenlogik untersucht werden soll, von anderen abgegrenzt werden kann. Stadt ist "zweifelsfrei eine genuin eigenständige Vergesellschaftungsform $" .{ }^{77}$ Stadt wird bezeichnet »als räumliche [...] Vergesellschaftungsform, deren distinkter Charakter in der raumstrukturellen Organisation von Dichte und großstädtischer Doxa liegt « ${ }^{78}$ Aber wenn man Wanne-Eickel und New York miteinander vergleicht, muss man dann nicht genauer sagen können, wo die »individuelle Gestalt « einer Stadt in RheinRuhr beziehungsweise Boswash beginnt und wo sie endet? ${ }^{79}$ Das Problem der Grenzziehung betrifft nicht nur die Stadt als siedlungsstrukturelle Kategorie. Das soziale, das ökonomische, das kulturelle, das politische, das infrastrukturelle System etc. einer Stadt, sie alle haben ihre unterschiedlichen Ausdehnungen. Und schließlich schafft sich jeder Bürger seine eigene Stadt. Mit der arbeitsteiligen Organisation des Alltags über verschiedene Gemeinden hinweg verwischen sich auch die wahrgenommenen Grenzen der Stadt, der sich der Bürger zugehörig fühlen könnte.

\section{Dichte}

Ähnlich verhält es sich mit dem dritten zentralen Begriff, dem der Dichte: Stadt »als raumstrukturelle Form der Verdichtung « zu definieren biete »den Vorteil, von allen

76 Manderscheid 2007, S. 55.

77 Berking 2008, S. 17.

78 Ebd., S. 29.

79 Dirksmeier 2011, S. 97 f. 
ebenso kontroversen wie arbiträren Versuchen einer sinhaltlichen Begriffsbestimmung zunächst einmal absehen zu können. Was, wie und wo und mit welchen Effekten verdichtet wird, sind dann die berüchtigten empirischen Fragen «. ${ }^{80}$ Aber sie müssten spätestens dann geklärt sein, wenn man sich in die Mühen der Feldforschung begeben will. Und gleich, wovon dann die Rede ist - von Bebauungs-Dichte, Funktions-Dichte, Bevölkerungs-Dichte oder Kommunikations-Dichte -, man wird daran nur insofern soziologische Aussagen knüpfen können, wie die spezifischen gesellschaftlichen Bedingungen untersucht sind, unter denen Dichte sich herstellt. Das Gewimmel auf der Karl-Marx-Allee, als diese noch Stalinallee hieß, hatte eine sehr andere soziale Bedeutung als unter Bedingungen von Markt und Demokratie.

\section{Perspektiven der Stadtforschung}

Nun ist es kein durchschlagender Einwand gegen ein Forschungsprogramm, dass es auf seine Fragen noch keine Antworten gefunden hat. Aber einige dieser Fragen sind nicht neu. ${ }^{81}$ »Wie Handlungs- und Wahrnehmungsmuster durch Bebauungsformen und Architekturen, durch geologische und klimatische Differenzen beeinflusst werden « ${ }^{82}$ ist eine Fragestellung, die von Montesquieu über Herder und Hegel bis zur Umweltpsychologie immer wieder thematisiert worden ist. »Die Unveränderlichkeit [...] der ganzen Beschaffenheit des Landes, in welchem eine Nation ihren bleibenden Wohnsitz hat, trägt zur Unveränderlichkeit des Charakters derselben bei. Eine Wüste, die Nachbarschaft des Meeres oder das Entferntsein vom Meere - alle diese Umstände können auf den Nationalcharakter Einfluß haben. «83 Aber trotzdem gibt es bisher keine belastbaren empirischen Erkenntnisse zu diesen Zusammenhängen. Müsste man sich nicht mit dieser Diskussion und vor allem mit den Gründen, weshalb sie bisher so wenig ertragreich war, etwas ausführlicher auseinandersetzen, bevor man sie zu einer der zentralen Fragen erhebt? Das Forschungsprogramm Eigenlogik stellt sich in den Rahmen der Wende zu Fragen des Raums und der Kultur (spatial und cultural turn) in der Soziologie. Aber man gewinnt zuweilen den Eindruck, mancher hätte diese Wendungen allzu rasant vollführt und vergessen, was vor der Wende lag.

Der cultural turn hat zu einer Fülle ethnographischer Studien geführt, die, wie die Chicagoer Schule ausgehend von einzelnen Orten, die dort angesiedelten Kulturen beschreiben (the Gold Coast and the slum) oder, ausgehend von bestimmten Milieus, verschiedene Orte in den Blick nehmen (the Hobo). Der wachsende Berg ethnographischer Studien im Rahmen von Urban Studies spiegelt die wachsende Bedeutung, die der Kultur in der internationalen Städtekonkurrenz zugeschrieben wird. Diese Studien vermitteln einen Eindruck von der überwältigenden Vielfalt der Stadt. Sie erzeugen ein Bild von Stadt als eines riesigen Warenhauses von Lebens-

80 Berking 2008, S. 22.

81 Dirksmeier 2011.

82 Löw 2008, S. 44.

83 Hegel, zitiert nach Garhammer 2000.

Leviathan, 41. Jg., 2/2013 
möglichkeiten. Man kann staunend davor stehen und bewundern, was die Stadt alles zu bieten hat. Aber die Welt der Stadt löst sich so auf in eine Vielzahl von Urban Villages, deren funktionale Zusammenhänge ebenso außerhalb des Gesichtsfelds bleiben wie die dahinter verborgenen Strukturen sozialer Ungleichheit. Letztere bedingen, dass die Stadt eben nicht allen alles bietet, sondern den einen die urbane Idylle und den anderen den Abfall des Urbanen. Es ist die Verbindung zwischen beidem, der kulturellen Vielfalt und der räumlichen, sozialen, politischen und ökonomischen Strukturen, die für die soziologische Stadtforschung relevant wäre.

Der Eigenlogik-Ansatz ist angetreten, um der soziologischen Stadtforschung die vergessenen Seiten ihres Gegenstands vorzuhalten. Dabei werden in der Freude über die Entdeckung bislang vernachlässigter Forschungsfelder die Bedeutung und die Neuheit dieser Themen manchmal überschätzt. Sieht man davon ab, so zeigen sich zumindest bei zwei Themen Überschneidungen mit der bisherigen soziologischen Stadtforschung. Das betrifft einmal die Annahme eigenlogischer Entwicklungen und zum anderen die Betonung materiell-physischer und kulturell-symbolischer Faktoren.

\section{Lokale und überlokale Determination}

Die These von der eigenlogischen Entwicklung jeder Stadt unterscheidet sich von der Oswald'schen These überlokaler Determination und lokaler Filterwirkung durch die Ausschließlichkeit, mit der singuläre, lokalgebundene Erklärungen in den Mittelpunkt gestellt werden, und durch das höhere Gewicht, das ihnen zugestanden wird. In der lokalen Politikforschung ist die Bedeutung der lokalen Ebene intensiv untersucht worden, nicht zuletzt deshalb, weil die Politikforschung sehr viel weniger Probleme als die Stadtsoziologie hat, das lokale System, dessen relatives Gewicht geklärt werden soll, zu definieren. Das lokale politisch-administrative System und die darauf bezogenen Akteursnetze sowie die Themen lokaler Politik sind weitgehend institutionell ausgeformt, die Stadt als Gegenstand der Soziologie hingegen kaum. Will man das Gewicht, das lokalen Faktoren bei der Erklärung von Stadtentwicklungsprozessen zukommt, untersuchen, sind allerdings Indikatoren notwendig, die die Gegenhypothesen nicht von vornherein ausschließen. Nimmt man die Häufigkeit, mit der Prinz-Heinrich-Mützen oder Trachtenjanker getragen werden, zum Indikator, dann werden Hamburg und München sich wahrscheinlich signifikant voneinander unterscheiden. Ähnliches dürfte auch für die Speisekarten in Kantinen herauskommen. Die Unterschiede zwischen den beiden Städten dürften hingegen sehr viel geringer ausfallen, wenn man den Zusammenhang von Dienstleistungsökonomie und Einkommenspolarisierung untersucht. Notwendig sind also vergleichende Forschungen, die sich auf relevante, also theoretisch begründete Indikatoren stützen. Wenn sich die Stadtforschung auf jene Gegenstände beschränken würde, bei deren Erklärung lokale Faktoren dominieren, würde sie politisch brisante und soziologisch relevante Themen ausklammern. 


\section{Die Stadt als physische und symbolische Struktur}

Die Kritik, die seitens der Vertreter des Darmstädter Ansatzes an der bisherigen soziologischen Stadtforschung geübt wird, betrifft neben der Unterschätzung eigenlogischer Entwicklungen die Vernachlässigung der physisch-materiell-technischen und der symbolischen Dimensionen der Stadt. Stefan Höhne hat zum Beispiel den städtischen Infrastrukturen geradezu demiurgische Qualitäten zugesprochen: »Sie prägen unsere Haltungen und unsere Ökonomie von Arbeit und Muße, unsere Wahrnehmung und unsere Körpergestalt, unsere Gefühle und unsere Wünsche. Sie prägen die Art und Weise, wie wir Freundschaften pflegen und Familien gründen, und die Art unserer Liebesbeziehungen und unserer Sexualität $" .{ }^{84}$ In der Stadtsoziologie ist die Rolle der städtischen Infrastrukturen für die Veränderungen der Lebensweisen zwar ebenfalls thematisiert worden, ${ }^{85}$ aber noch längst nicht ausreichend. Infrastrukturdisparitäten können zusammen mit der Selektivität von (Bildungs-)Institutionen und dem örtlichen Angebot an Arbeitsplätzen Lebenschancen beeinflussen, ebenso wie mit dem Auf- und Abstieg von Städten unterschiedliche Chancen für den Auf- und Abstieg von Individuen verbunden sind. Löw ${ }^{86}$ hat recht, wenn sie schreibt: »Die Forschung zur Eigenlogik der Städte kann die soziale Ungleichheitsforschung um eine weitere Perspektive ergänzen: die unterschiedliche Verteilung von Lebenschancen je nach Stadt, gebunden an die jeweiligen städtischen Praxisformen «. Ebenso zu Recht betont Löw, dass in der Stadtforschung die Art und Weise, wie sich »Menschen die scheinbaren Tatsachen wiederum deutend aneignen ", also die subjektive Konstitution von Sinn eine wesentliche Rolle spielen sollte. Die Forderung, die subjektive Konstitution des Gegenstands Stadt in die Untersuchung systematisch aufzunehmen, ist zwar schon früh erhoben worden, ${ }^{87}$ aber bisher sind entsprechende Studien selten. Ähnlich benennt Friedrichs ${ }^{88}$ als zentrales Thema der Stadtforschung die Untersuchung der Diversität jeweils unterschiedlich wahrgenommener »Opportunitätsstrukturen «, die mit lokal unterschiedlichen Arbeits- und Wohnungsmärkten, Bevölkerungsstrukturen, Infrastrukturen und Umweltbedingungen zusammenhängen.

Historisch gewachsene Machtstrukturen, die Eigentumsverhältnisse an Grund und Boden, die örtliche Wirtschafts- und Sozialstruktur, die historisch vermittelte Imprägnierung verschiedener Orte mit verschiedenen Labels und Bildern und die Wahrnehmung dieser Gegebenheiten durch verschiedene soziale Gruppen, all das kann einen Unterschied machen. Lokalität macht einen Unterschied, aber welchen genau und für wen ist noch keineswegs hinreichend geklärt. Seit Längerem schon wird im Zusammenhang von Nachbarschafts- oder Kontexteffekten auch die Rolle

84 » They shape our habits and economics of work and leisure, our perceptions and bodies, and our affects and desire. They shape the way we realize friendships and family and the way we realize love relations and sexuality. " (Höhne 2012, S. 149; Übersetzung W.S.)

85 Häußermann, Siebel 1996; Häußermann, Siebel 2004.

86 Löw 2011, S. 64.

87 Krämer-Badoni 1992

88 Friedrichs 2011, S. 37 f.

Leviathan, 41. Jg., 2/2013 
lokal unterschiedlicher physisch-materieller, kultureller, sozialer und symbolischer Bedingungen diskutiert, ${ }^{89}$ ohne dass hier schon genügend empirisch belastbare Ergebnisse vorlägen. Die Hannoveraner Milieustudien ${ }^{90}$ könnten dabei ein Modell bieten für eine gelungene Verknüpfung von strukturellen mit konstruktivistischen Ansätzen in der Stadtforschung. Und solche Studien müssen eingebettet sein in eine systematisch vergleichende Stadtforschung, also mehr bieten als "sinnrekonstruktive Einzelfallanalyse.$^{91}$

Der Darmstädter Ansatz ordnet sich ein in die kulturalistische Wende, den cultural turn der Sozialwissenschaften. Damit ist nicht nur die subjektive Konstitution sozialer Wirklichkeit gemeint, sondern die Betonung kultureller Faktoren bei der Erklärung gesellschaftlichen Wandels und generell eine umfassende "Kulturalisierung " von Gesellschaft, wie sie Reckwitz ${ }^{92}$ mit seiner These von einem alle gesellschaftlichen Bereiche durchziehenden »Kreativitätsdispositiv « behauptet. In der Stadtsoziologie haben diese Fragen erst seit den 1990er Jahren wieder mehr Beachtung gefunden: in der Lebensstil- und Milieuforschung, den Untersuchungen zur »Festivalisierung « und »Kulturalisierung " der Stadt und Regionalpolitik ${ }^{93}$ und zum Wandel städtischer Ökonomien zur Symbolproduktion. ${ }^{94}$ Seit einiger Zeit haben diese Veränderungen unter dem Schlagwort »Creative Cities «95 auch Relevanz in der Stadtpolitik gewonnen. Die Unterschiede, mit der diese Themen in der Stadtsoziologie bearbeitet werden, liegen hier wiederum eher in einem vornehmlich nomothetischen Interesse einerseits, einem vornehmlich ideographischen andererseits. Es scheint, als träten im gegenwärtigen Streit innerhalb der Stadtsoziologie die beiden Perspektiven auf Stadt gegeneinander an, mit denen die empirische Soziologie der Stadt am Anfang des vorigen Jahrhunderts in Chicago begonnen hatte: Ernest Burgess stand für einen Blick auf die Stadt, der darauf gerichtet war, aus der Beschreibung und Erklärung sozialräumlicher Strukturen und deren Veränderungen verallgemeinerbare Gesetzmäßigkeiten städtischer Entwicklung zu gewinnen. Robert Park und seine Schüler dagegen waren fasziniert von der Vielfalt der Orte und der Lebensweisen, die sie in Chicago vorfanden und die sie in einer Vielzahl von qualitativen Fallstudien dokumentiert haben. Eine künftige Soziologie der Stadt könnte versuchen, diese beiden Perspektiven, die seitdem weit auseinandergetreten waren, wieder miteinander zu verbinden. Notwendig wären dazu theoretisch angeleitete und in Strukturanalysen eingebettete Fallstudien, deren Erträge durch systematische Vergleiche und theoretische Einordnung zu verallgemeinerbaren Aussagen verdichtet werden. Der schon erwähnte Herbert Gans hat mit seinen »Urban Villagers « eine Studie vorgelegt, die mit ihrer Verbindung von struktureller Analyse, qualita-

89 Häußermann 2000; Farwick 2001.

90 Vester et al. 2001.

91 Berking, Löw 2005, S. 12.

92 Reckwitz 2012.

93 Häußermann, Siebel 1993 a; Häußermann, Siebel 1993 b.

94 Zukin 1995.

95 Florida 2002. 
tiver Fallstudie und Rückbindung in die soziologische Theorietradition exemplarisch sein könnte für eine Stadtsoziologie, die die Aufspaltung der beiden Perspektiven auf Stadt, wie sie in der Chicagoer Schule präsent waren, überwindet.

Ähnlich wäre im Hinblick auf die Eigenlogik-Thesen des Darmstädter Ansatzes zu argumentieren. Auch Sigmund Freud hat eine Theorie der Eigenlogik entwickelt, der Eigenlogik des Psychischen. Aber die Psychoanalyse als eine Theorie, die besonders entschieden die Singularität jedes Individuums betont, ist zugleich eine Theorie mit besonders starken Annahmen zu einer verallgemeinerbaren Struktur des psychischen Prozesses. Adorno hat auf das Paradox hingewiesen, dass Freud im Innersten der Psyche auf Gesellschaftliches gestoßen sei - das Über-Ich -, und Norbert Elias hat die historische Entstehung dieses Über-Ichs herausgearbeitet. Auch im Innersten der Stadt stößt man auf Gesellschaftliches: die Eigentumsverhältnisse, die Trennung von Wohnen und Arbeiten, die Polarisierung von Öffentlichkeit und Privatheit, soziale Ungleichheit und Machtverhältnisse, den Wandel der Rolle der Frau ... Und umgekehrt spielt im Innersten von moderner Gesellschaft Stadt eine wesentliche Rolle: Sozialer Wandel wäre nicht zu verstehen ohne den Prozess der Urbanisierung. Seit Psychoanalyse und Soziologie existieren, geht es um die Frage der Vermittlung von Individuum und Gesellschaft. Solche Vermittlung ist nicht als Addition zu konzipieren, sondern als dialektischer Prozess. Dasselbe gilt für die Vermittlung eigenlogischer städtischer Entwicklungen mit Prozessen des gesellschaftlichen Strukturwandels.

Ich kann also keine Alternative erkennen, wonach der Darmstädter Ansatz an die Stelle der bisherigen Stadtsoziologie treten könnte. Es geht um die Vermittlung dessen, was sich die beiden Ansätze als die jeweils vom anderen vergessenen Seiten der Stadt als einer höchst widersprüchlichen Realität vorhalten. Häußermann ${ }^{96}$ hatte ans Ende seiner Rezension ein Zitat von Max Weber gestellt. Ich wiederhole es hier: Es kann »natürlich nicht die Absicht sein, anstelle einer einseitig smaterialistischen< eine ebenso einseitige spiritualistische kausale Kultur- und Geschichtsdeutung zu setzen. Beide sind gleich möglich, aber mit beiden ist, wenn sie nicht Vorarbeit, sondern Abschluss der Untersuchung zu sein beanspruchen, der historischen Wahrheit gleich wenig gedient «.

\section{Literatur}

Bagnasco, Arnaldo; Le Galès, Patrick 2000. »European societies as collective actors? «, in Cities in contemporary Europe, hrsg. v. Bagnasco, Arnaldo; Le Galès, Patrick, S. 1-32. Cambridge: Cambridge University Press.

Bahrdt, Hans-Paul 1998. Die moderne Großstadt. Opladen: Leske + Budrich.

BBSR (Bundesinstitut für Bau-, Stadt- und Raumforschung) 2011. »Mehrfachverkäufe von Mietwohnungsbeständen - Forschungen Heft 146«, in Informationen aus der Forschung des BBSR 2, S. 4.

Berking, Helmuth 2008. " Städte lassen sich an ihrem Gang erkennen wie Menschen< - Skizzen zur Erforschung der Stadt und der Städte", in Die Eigenlogik der Städte, hrsg. v. Berking, Helmuth; Löw, Martina, S. 15-31. Frankfurt a. M.: Campus.

96 Häußermann 2011, S. 170 f.

Leviathan, 41. Jg., 2/2013 
Berking, Helmuth; Löw, Martina. Hrsg. 2005. Die Wirklichkeit der Städte. Soziale Welt, Sonderband 16. Baden-Baden: Nomos.

Berking, Helmuth; Löw, Martina. Hrsg. 2008. Die Eigenlogik der Städte. Frankfurt a. M.: Campus.

Bockrath, Franz 2008. »Städtischer Habitus - Habitus der Stadt «, in Die Eigenlogik der Städte, hrsg. v. Berking, Helmuth; Löw, Martina, S. 55-82. Frankfurt a. M.: Campus.

Croon, Helmut; Utermann, Kurt 1958. Zeche und Gemeinde. Untersuchungen über den Strukturwandel einer Zechengemeinde im nördlichen Ruhrgebiet. Tübingen: Mohr.

Dirksmeier, Peter 2011. "Argumentationslinien der Klassischen Geographie in der neueren deutschsprachigen Stadtsoziologie ", in Lokalistische Stadtforschung, kulturalisierte StädteZur Kritik einer Eigenlogik der Städte, hrsg. v. Kemper, Jan; Vogelpohl, Anne, S. 88-104. Münster: Westfälisches Dampfboot.

Elias, Norbert 1976. Über den Prozeß der Zivilisation. Frankfurt a. M.: Suhrkamp.

Engels, Friedrich 1959 [1845]: »Die Lage der arbeitenden Klasse in England «, in $M E W$, Band 2, S. 256-305. Berlin-Ost: Dietz.

Farwick, Andreas 2001. Segregierte Armut in der Stadt. Opladen: Leske + Budrich.

Florida, Richard 2002. The rise of the creative class. New York: Basic Books.

Friedrichs, Jürgen 2011. "Das Städtische und die Stadtsoziologie ", in Die Besonderheit des Städtischen, hrsg. v. Herrmann, Heike et al., S. 33-47. Wiesbaden: VS Verlag für Sozialwissenschaften, S. 33-47.

Gans, Herbert 1962. The urban villagers: group and class in the life of Italian-Americans. New York et al.: Free Press.

Garhammer, Manfred 2000: »Die Bedeutung des Raums für die regionale, nationale und globale Vergesellschaftung - zur Aktualität von Simmels Soziologie des Raums ", in Kultur und Region im Zeichen der Globalisierung: Wohin treiben die Regionalkulturen? Beiträge zum 14. Interdisziplinären Kolloquium des Zentralinstituts, hrsg. von Bahadir, Sefik A., S. 15-39. Neustadt: Degener.

Gestring, Norbert 2011. »Habitus, Handeln, Stadt - Eine soziologische Kritik der `Eigenlogik der Städte " in Lokalistische Stadtforschung, kulturalisierte Städte - Zur Kritik einer Eigenlogik der Städte, hrsg. v. Kemper, Jan; Vogelpohl, Anne, S. 40-53. Münster: Westfälisches Dampfboot.

Goebel, Jan; Gornig, Martin; Häußermann, Hartmut 2012. »Wirtschaftliche Dynamik der Städte und Einkommenspolarisierung ", in Leviathan 40, 3, S. 371-398.

Grubbauer, Monika 2012. »Architektur und Stadt: Anmerkungen aus politisch-ökonomischer Perspektive", in geographische revue 14, 1, S. 59-73.

Hasse, Jürgen 2008. »Stadt ‘ als schwimmender Terminus «, in Die Eigenlogik der Städte, hrsg. v. Berking, Helmuth; Löw, Martina, S. 313-334. Frankfurt a. M.: Campus.

Häußermann, Hartmut 2000. »Die Krise der `sozialen Stadt`«, in Aus Politik und Zeitgeschichte B10-11, S. 13-21.

Häußermann, Hartmut 2011. » Soziologie der Städte‘. Eine Rezension «, in Lokalistische Stadtforschung, kulturalisierte Städte - Zur Kritik einer Eigenlogik der Städte, hrsg. v. Kemper, Jan; Vogelpohl, Anne, S. 158-171. Münster: Westfälisches Dampfboot.

Häußermann, Hartmut; Siebel, Walter 1986. „Die Polarisierung der Großstadtentwicklung im Süd-Nord-Gefälle", in Süd-Nord-Gefälle in der Bundesrepublik?, hrsg. v. Friedrichs, Jürgen; Häußermann, Hartmut; Siebel, Walter, S. 70-96. Opladen: Westdeutscher Verlag.

Häußermann, Hartmut; Siebel, Walter. Hrsg. 1993 a. Festivalisierung der Stadtpolitik. Leviathan, Sonderheft 13. Opladen: Westdeutscher Verlag.

Häußermann, Hartmut; Siebel, Walter 1993 b. »Die Kulturalisierung der Regionalpolitik «, in Geographische Rundschau 45, 4, S. 218-223.

Häußermann, Hartmut; Siebel, Walter 1996. Soziologie des Wohnens. Weinheim: Juventa.

Häußermann, Hartmut; Siebel, Walter 2004. Stadtsoziologie. Frankfurt a. M.: Campus.

Höhne, Stefan 2012. "An endless flow of machines to serve the city: infrastructural assemblages and the quest for the metropolis ", in Thick space, hrsg. v. Brantz, Dorothee; Disko, Sasha; Wagner-Kyora, Georg, S. 141-164. Bielefeld: transcript.

Kemper, Jan; Vogelpohl, Anne. Hrsg. 2011. Lokalistische Stadtforschung, kulturalisierte StädteZur Kritik einer Eigenlogik der Städte. Münster: Westfälisches Dampfboot.

König, Renè 1958. Grundformen der Gesellschaft: Die Gemeinde. Hamburg: Rowohlt. 
Koll-Schretzenmayr, Martina; Ritterhoff, Frank; Siebel, Walter 2009. »In quest of the good urban life «, in Urban Studies 46, 13, S. 2731-2747.

Krämer-Badoni, Thomas 1992. "Die Stadt als sozialwissenschaftlicher Gegenstand «, in Stadt und Raum. Soziologische Analysen, hrsg. v. Häußermann, Hartmut et al., S. 1-29. Pfaffenweiler: Centaurus.

Kronauer, Martin 2002. Exklusion - Die Gefährdung des Sozialen im hoch entwickelten Kapitalismus. Frankfurt a. M.: Campus.

Läpple, Dieter 2005. »Phönix aus der Asche: Die Neuerfindung der Stadt «, in Die Wirklichkeit der Städte. Soziale Welt, Sonderband 16, hrsg. v. Berking, Helmuth; Löw, Martina, S. 397-413. Baden-Baden: Nomos.

Löw, Martina. Hrsg. 2002. Differenzierungen des Städtischen. Opladen: Leske + Budrich.

Löw, Martina 2008. »Eigenlogische Strukturen «, in Die Eigenlogik der Städte, hrsg. v. Berking, Helmuth; Löw, Martina, S. 33-53. Frankfurt a. M.: Campus.

Löw, Martina 2010. Soziologie der Städte. Berlin: Suhrkamp.

Löw, Martina 2011. "Städte als unterscheidende Erfahrungsräume ", in Die Besonderheit des Städtischen, hrsg. v. Herrmann, Heike et al., S. 49-67. Wiesbaden: VS Verlag für Sozialwissenschaften.

Löw, Martina; Noller, Peter 2010. »Eine Insel der Glückseligen «, in Typisch Darmstadt, hrsg. v. Löw, Martina; Noller, Peter; Süß, Sabine, S. 265-273. Frankfurt a. M.: Campus.

Löw, Martina; Noller, Peter; Süß, Sabine 2010: »Einleitung «, in Typisch Darmstadt, hrsg. v. Löw, Martina; Noller, Peter; Süß, Sabine, S. 9-14. Frankfurt a. M.: Campus.

Lynch, Kevin o. J. Das Bild der Stadt. Bawwelt Fundamente 16. Berlin: Ullstein.

Manderscheid, Katharina 2007. »Urbanität im 21. Jahrhundert", in Die Stadt in der Sozialen Arbeit, hrsg. v. Baum, Detlef, S. 52-70. Wiesbaden: VS Verlag für Sozialwissenschaften.

Marx, Karl; Engels, Friedrich 1980 [1848]. »Manifest der Kommunistischen Partei «, in MEW, Band 4, S. 459-493. Berlin-Ost: Dietz.

Mönninger, Michael 2012. »Ankunftsstädte, Abschiedsstädte oder: Lernen von Mumbai. Warum westliche Planer sich für Mega-Cities interessieren «, in geographische revue 14, 1, S. 43-57.

Münch, Richard 1993. Das Projekt Europa. Zwischen Nationalstaat, regionaler Autonomie und Weltgesellschaft. Frankfurt a. M.: Suhrkamp.

Münch, Richard 1996. Europäische Identitätsbildung: Zwischen globaler Dynamik, nationaler und regionaler Gegenbewegung. Antrittsvorlesung vom 14. Februar 1996 an der Universität Bamberg.

Nassehi, Armin 2002. »Dichte Räume. Städte als Synchronisations- und Inklusionsmaschinen ", in Differenzierungen des Städtischen, hrsg. v. Löw, Martina, S. 211-232. Opladen: Leske + Budrich.

Oswald, Hans 1966. Die überschätzte Stadt. Olten: Walter.

Reckwitz, Andreas 2012: Die Erfindung der Kreativität. Frankfurt a. M.: Suhrkamp.

Rodenstein, Marianne 2008. »Die Eigenart der Städte - Frankfurt und Hamburg im Vergleich « in Die Eigenlogik der Städte, hrsg. v. Berking, Helmuth; Löw, Martina, S. 261-311. Frankfurt a. M.: Campus.

Schäfers, Bernhard 2011. »Besprechung von Martina Löw, Soziologie der Städte", in Kölner Zeitschrift für Soziologie und Sozialpsychologie 63, S. 513-515.

Siebel, Walter 1974. Entwicklungstendenzen kommunaler Planung. Schriftenreihe des Bundesministers für Raumordnung, Bauwesen und Städtebau: Städtebauliche Forschung 03.028. Bonn-Bad Godesberg.

Siebel, Walter 2004. "Einleitung «, in Die europäische Stadt, hrsg. v. Siebel, Walter, S. 11-50. Frankfurt a. M.: Suhrkamp.

Siebel, Walter 2010. »Voraussetzungen gelingender Integration und die Aufgaben der Integrationspolitik ", in In diesem Geschäft gibt es keine Mathematik: 20 Jahre Schader-Stiftung, hrsg. v. der Schader-Stiftung, S. 212-226. Darmstadt: Schader-Stiftung.

Siebel, Walter 2012. »Die europäische Stadt", in Handbuch Stadtsoziologie, hrsg. v. Eckardt, Frank, S. 201-211. Wiesbaden: Springer VS.

Simmel, Georg 1989. Philosophie des Geldes. Gesamtausgabe, Band 6, hrsg. v. Rammstedt, Otthein. Frankfurt a. M.: Suhrkamp.

Steinert, Heinz 2010. Max Webers unwiderlegbare Fehlkonstruktionen. Die protestantische Ethik und der Geist des Kapitalismus. Frankfurt a. M.: Campus.

Leviathan, 41. Jg., 2/2013 
Stichweh, Rudolf 2000. "Raum, Region und Stadt in der Systemtheorie " in Rudolf Stichweh: Die Weltgesellschaft. Soziologische Analysen, S. 184-206. Frankfurt a. M.: Suhrkamp.

Taylor, Jan; Evans, Karen; Fraser, Penny 1996. Tale of two cities. Global change, local feeling and everyday life in the North of England. London: Routledge.

Vester, Michael et al. 2001. Soziale Milieus im gesellschaftlichen Strukturwandel. Zwischen Integration und Ausgrenzung. Frankfurt a. M.: Suhrkamp.

Wollmann, Hellmut 2013. »Öffentliche Dienstleistungen zwischen öffentlich/munizipalem und privatem Sektor. >Comeback der Kommunen? ", in Stadtgesellschaft, hrsg. v. Kronauer, Martin; Siebel, Walter. Frankfurt a. M.: Campus (im Erscheinen).

Zimmermann, Karsten 2008: »Eigenlogik der Städte - eine politikwissenschaftliche Sicht «, in Die Eigenlogik der Städte, hrsg. v. Berking, Helmuth; Löw, Martina, S. 207-230. Frankfurt a. M.: Campus.

Zukin, Sharon 1995. The cultures of cities. Oxford: Blackwell. 
Zusammenfassung: Der Beitrag beginnt mit einer Diskussion der Unterschiede zwischen dem Darmstädter Ansatz der Eigenlogik der Städte und der soziologischen Stadtforschung. Anschließend wird danach gefragt, ob der Anspruch, die Soziologie der Stadt neu zu gründen, eingelöst wird. Danach werden einige Fragen benannt, die auch bei einem bescheideneren Anspruch im Rahmen des Ansatzes der Eigenlogik-Forschung beantwortet werden müssen. Zum Schluss wird versucht, einige Perspektiven einer die Anregungen des Darmstädter Ansatzes aufnehmenden soziologischen Stadtforschung zu formulieren.

Stichworte: Stadtsoziologie, kulturalistische Wende

\section{City, inequality and diversity}

Summary: The article begins with a discussion of the differences between urban sociology and the "Darmstadt Approach " to the intrinsic logik of the city. This is followed by an examination of whether the claim to refound urban sociology has been redeemed. Thereafter questions are formulated which need to be answered, even when the claim to found a new approach to the study of the urban is not upheld. The article concludes with some research perspectives.

Keywords: urban sociology, urban research, cultural turn

\section{Autor}

Prof. Dr. Walter Siebel

Institut für Soziologie

Carl von Ossietzky Universität

26111 Oldenburg

Leviathan, 41. Jg., 2/2013 